\title{
COMUNICAÇÃO
}

\section{EFICIÊNCIA DE PRODUTOS TERMONEBULÍGENOS NO CONTROLE DE Atta laevigata (Hymenoptera: Formicidae) EM PLANTIO DE EUCALIPTO}

\author{
Efficiency of products for thermonebulization on the control of Atta laevigata \\ (Hymenoptera: Formicidae) in eucalypus plantations \\ Ronald Zanetti ${ }^{1}$, José Cola Zanuncio ${ }^{2}$, Alan Souza-Silva ${ }^{3}$, Lúcia Aparecida Mendonça ${ }^{3}$, \\ Jorge Olavo Souza Mattos ${ }^{4}$, Muriel Santos Rizental ${ }^{5}$
}

\begin{abstract}
RESUMO
Avaliou-se a eficiência de produtos termonebulígenos, a base de clorpirifós ou de extratos vegetais, comparativamente ao uso de isca formicida, a base de sulfluramida, no controle de Atta laevigata (F. Smith, 1858) (Hymenoptera: Formicidae). Foi avaliada a percentagem de paralisação das atividades de corte de folhas e de movimentação de formigas de A. laevigata aos três, $12,36,63$ e 86 dias após a aplicação dos tratamentos. Na última avaliação, os formigueiros foram abertos para a verificação da eficiência de controle. Todos os produtos testados apresentaram alta percentagem de paralisação das atividades de corte e de movimentação das formigas aos três e 12 dias após a aplicação, respectivamente. Os produtos a base de clorpirifós e um a base de extratos vegetais apresentaram alta eficiência no controle de $A$. laevigata, sendo mais efetivos que a isca formicida testada.
\end{abstract}

Termos para indexação: Termonebulização, formigas cortadeiras, Eucalyptus, praga.

\section{ABSTRACT}

The efficiency of products formulated with chlorpyrifos or plants extracts in thermonebuzation was evaluated and compared to the use of the granulated bait formulated with sulfluramid in the control of Atta laevigata (F. Smith, 1858) (Hymenoptera: Formicidae). The cutting activity and the movement of ants on the colonies were evaluated at three, 12, 36, 63 and 86 days after the application of the treatments. The colonies of these ants were excavated in the last evaluation to obtain the efficiency of each product. All products stopped the cutting activity and movements of the individuals of A. laevigata three and 12 days after their application, respectively. Products formulated with chlorpyrifos and one with plant extracts were more efficient than granulated bait.

Index terms: Thermonebulization, leaf-cutting ants, eucalyptus, control.

(Recebido em 19 de agosto de 2005 e aprovado em 15 de setembro de 2006)

As formigas cortadeiras do gênero Atta estão incluídas entre os principais insetos-praga de cultivos no Brasil (CRUZ et al., 1996). Esses insetos causam desfolha em vários tipos de plantas, como eucalipto, gramíneas e plantas nativas, utilizando as folhas para cultivar o fungo, que é empregado na sua alimentação (ZANUNCIO et al., 2002).

O controle dessas formigas em áreas cultivadas com essências florestais tem sido feito, basicamente, com iscas granuladas, por ser um método prático e econômico em comparação a outros métodos de controle (DELLA LUCIA \& VILELA, 1993). No entanto, a termonebulização pode ser empregada como alternativa ao uso dessas iscas, principalmente em períodos chuvosos (CRUZ et al., 1984). A termonebulização pode apresentar alta eficiência para formigueiros grandes, especialmente nas operações de combate inicial, durante a implantação de florestas cultivadas (ZANETTI et al., 2002). Além disso, esse método de controle pode ser utilizado em qualquer época do ano, em terrenos encharcados ou secos e não requer o preparo e medição dos formigueiros (ANJOS et al., 1998).

O objetivo desse trabalho foi avaliar a paralisação de movimentação das formigas e de corte de folhas e a

\footnotetext{
'Engenheiro Florestal, Doutor em Ciências Florestais, Professor - Departamento de Entomologia/DEN - Universidade Federal de Lavras/UFLA - Cx. P. 3037 - 37200-000 - Lavras, MG - zanetti@ufla.br

²Engenheiro Florestal, PhD., Professor - Departamento de Biologia/DBA - Universidade Federal de Viçosa/UFV - Campus Universitário - 36571-000 Viçosa, MG - zanuncio@ufv.br

${ }^{3}$ Engenheiros Agrônomos, Doutores em Entomologia - Departamento de Entomologia/DEN - Universidade Federal de Lavras/UFLA - Cx. P. 3037 37200-000 - Lavras, MG - lucia@ufla.br

${ }^{4}$ Graduando em Agronomia - Departamento de Entomologia/DEN - Universidade Federal de Lavras/UFLA - Cx. P. 3037 - $37200-000$ - Lavras, MG

${ }^{5}$ Agrônoma - Departamento de Entomologia/DEN - Universidade Federal de Lavras/UFLA - Cx. P. 3037 - 37200-000 - Lavras, MG - rizental@ufla.br
} 
eficiência de controle de produtos termonebulígenos, diluídos em querosene ou óleo diesel, contra A. laevigata em áreas cultivadas com eucalipto.

Foram selecionados 64 formigueiros de Atta laevigata (F. Smith, 1858) (Hymenoptera: Formicidae) em um eucaliptal, no município de Três Marias, MG, em 2000. Esses formigueiros foram distribuídos aleatoriamente em oito tratamentos (Tabela 1).

A termonebulização foi realizada em cada ninho com um aparelho Puls-Fog, sendo o produto aplicado em um olheiro ativo do sauveiro até a saturação do formigueiro, sendo os demais olheiros tampados na medida em que a fumaça saia deles. A isca formicida foi aplicada numa dosagem de $8 \mathrm{~g} / \mathrm{m}^{2}$ de área de terra solta do ninho, próxima aos olheiros ativos.

As avaliações foram feitas aos três, $12,36,63$ e 86 dias após a aplicação dos produtos (DAA). Em cada avaliação, foi medida a percentagem de ninhos com atividade de movimentação de formigas, por meio de observação direta ou efetuando-se perturbações nos olheiros com uma vara de madeira para verificar essa possível atividade. Mediu-se, também, a percentagem de ninhos com paralisação da atividade de corte de folhas pelas formigas, observando-se a presença de folhas recentemente cortadas nas trilhas, nos olheiros de alimentação ou sobre os montes de terra solta dos formigueiros. Na última avaliação (86 DAA), mediu-se a percentagem de formigueiros mortos (eficiência de controle), por meio da escavação dos ninhos.

Todos os produtos termonebulígenos e a isca formicida testada apresentaram alta percentagem de paralisação da atividade de corte por Atta laevigata nos diferentes dias após a aplicação (Tukey, $\mathrm{p}<0,05$ ), com valores médios de $97,5 \%$ e $100 \%$ (Tabela 2), sendo essa paralisação rápida (três dias após a aplicação), independente do produto testado.

Tabela 1 - Tratamentos usados no teste de eficiência de produtos termonebulígenos contra formigas cortadeiras. Três Marias, Minas Gerais (2000).

\begin{tabular}{lcccl}
\hline \multicolumn{1}{c}{ Nome técnico } & Nome comercial & Formulação & Concentração & Dose do produto \\
\hline Clorpirifós & Dursban & $\mathrm{CE}$ & $480 \mathrm{~g} / \mathrm{litro}$ & $100 \mathrm{ml} / 1$ de diesel \\
Clorpirifós & Dursban & $\mathrm{CE}$ & $480 \mathrm{~g} / \mathrm{litro}$ & $100 \mathrm{ml} / 1$ de querosene \\
Clorpirifós & Dragnet & $\mathrm{CE}$ & $480 \mathrm{~g} / \mathrm{litro}$ & $100 \mathrm{ml} / 1$ de diesel \\
Clorpirifós & Dragnet & $\mathrm{CE}$ & $480 \mathrm{~g} / \mathrm{litro}$ & $100 \mathrm{ml} / 1 \mathrm{de}$ querosene \\
Extratos vegetais & Attacid A & $\mathrm{CE}$ & - & $200 \mathrm{ml} / 1 \mathrm{de}$ diesel \\
Extratos vegetais & Attacid B & $\mathrm{CE}$ & - & $200 \mathrm{ml} / 1$ de querosene \\
Sulfluramida & Mirex-S MAX & $\mathrm{G}$ & $3 \mathrm{~g} / \mathrm{kg}$ & $8 \mathrm{~g} / \mathrm{m}^{2}$ de formigueiro \\
\hline
\end{tabular}

Tabela 2 - Percentagem de paralisação da atividade de corte por Atta laevigata em diferentes dias após a aplicação (DAA) de produtos termonebulígenos e da isca formicida. Três Marias, Minas Gerais (2000).

\begin{tabular}{|c|c|c|c|c|c|c|}
\hline \multirow{2}{*}{ Tratamento } & \multicolumn{5}{|c|}{ DAA } & \multirow{2}{*}{ Média } \\
\hline & 3 & 12 & 36 & 63 & 86 & \\
\hline Testemunha & $0,0 \mathrm{~b}$ & $0,0 \mathrm{~b}$ & $0,0 \mathrm{~b}$ & $0,0 \mathrm{~b}$ & $0,0 \mathrm{~b}$ & $0,0 \mathrm{~b}$ \\
\hline Dursban CE a $100 \mathrm{ml} /$ litro de diesel & $100,0 \mathrm{a}$ & $87,5 \mathrm{a}$ & 100,0 a & $100,0 \mathrm{a}$ & 100,0 a & $97,5 \mathrm{a}$ \\
\hline Dursban CE a $100 \mathrm{ml} /$ litro de querosene & $100,0 \mathrm{a}$ & $100,0 \mathrm{a}$ & $100,0 \mathrm{a}$ & $87,5 \mathrm{a}$ & $100,0 \mathrm{a}$ & $97,5 \mathrm{a}$ \\
\hline Dragnet CE a $140 \mathrm{ml} /$ litro de diesel & $100,0 \mathrm{a}$ & $100,0 \mathrm{a}$ & 100,0 a & $100,0 \mathrm{a}$ & $100,0 \mathrm{a}$ & $100,0 \mathrm{a}$ \\
\hline Dragnet CE a $140 \mathrm{ml} /$ litro de querosene & $100,0 \mathrm{a}$ & $100,0 \mathrm{a}$ & $100,0 \mathrm{a}$ & $87,5 \mathrm{a}$ & $100,0 \mathrm{a}$ & $97,5 \mathrm{a}$ \\
\hline Attacid A a $200 \mathrm{ml} /$ litro de diesel & $87,5 \mathrm{a}$ & $100,0 \mathrm{a}$ & $100,0 \mathrm{a}$ & $100,0 \mathrm{a}$ & $100,0 \mathrm{a}$ & $100,0 \mathrm{a}$ \\
\hline Attacid B a $200 \mathrm{ml} /$ litro de diesel & $100,0 \mathrm{a}$ & $100,0 \mathrm{a}$ & $100,0 \mathrm{a}$ & $87,5 \mathrm{a}$ & $100,0 \mathrm{a}$ & $97,5 \mathrm{a}$ \\
\hline Mirex-S a $8 \mathrm{~g} / \mathrm{m}^{2}$ de formigueiro & $100,0 \mathrm{a}$ & $100,0 \mathrm{a}$ & $100,0 \mathrm{a}$ & $100,0 \mathrm{a}$ & $100,0 \mathrm{a}$ & $100,0 \mathrm{a}$ \\
\hline
\end{tabular}

Médias seguidas pela mesma letra na coluna não diferem entre si (Tukey, p>0,05). 
A rapidez de paralisação de corte é critério fundamental para a seleção de produtos formicidas, pois é essencial evitar danos às plantas cultivadas, principalmente na fase jovem, quando essas plantas estão mais suscetíveis. Essa paralisação, em formigueiros tratados com termonebulígenos é imediata (ANJOS et al., 1998), semelhante ao verificado nesse estudo. No caso das iscas formicidas, estudos relatam a paralisação de corte após seis dias da aplicação para Atta laevigata (ALVES et al., 1996, 1997; ZANUNCIO et al., 1992) e Atta sexdens rubropilosa (LARANJEIRO \& ZANUNCIO, 1995;ZANETTI et al., 2003; ZANUNCIO et al., 2002). No entanto, a isca testada nesse estudo apresentou tempo de paralisação de corte semelhante aos termonebulígenos.

Quase todos os tratamentos paralisaram as atividades de movimentação de formigas, na maioria dos formigueiros tratados, a partir dos três DAA, atingindo o máximo aos 12 DAA. Dragnet CE a $140 \mathrm{ml} / \mathrm{l}$ de querosene se destacou entre os produtos testados por apresentar elevada percentagem de paralisação de movimentação de formigas em todos os dias de avaliação (Tabela 3), enquanto os demais produtos tiveram variação dessa paralisação no tempo. Os formigueiros tratados com Dursban CE a 140 $\mathrm{ml} / \mathrm{l}$ de diesel apresentaram menor percentagem de paralisação aos 36 DAA, enquanto Dursban CE a 100 ml/l de querosene, Attacid A a $200 \mathrm{ml} / \mathrm{l}$ de diesel, Attacid B a $200 \mathrm{ml} / \mathrm{l}$ de diesel e Mirex-S Max a 8g/m² tiveram o mesmo comportamento aos 63 DAA.

Os produtos Dursban CE a $100 \mathrm{ml} / \mathrm{l}$ diesel ou querosene, Dragnet CE a $140 \mathrm{ml} / \mathrm{l}$ de diesel ou querosene e
Attacid A a 200 ml/l de diesel apresentaram alta eficiência no controle de A. laevigata. Os produtos Attacid B a 200 $\mathrm{ml} / 1$ de diesel e Mirex-S Max a $8 \mathrm{~g} / \mathrm{m}^{2}$ apresentaram eficiência de controle menor que os demais, porém diferentes e superiores à testemunha (Tabela 3). Resultados semelhantes foram obtidos por Gomes et al. (1989) com produtos termonebulígenos à base clorpirifós, que apresentaram eficiência de $100 \%$ no controle de Atta spp. Santos et al. (2007) encontraram eficiência média de $75 \%$ no controle de Atta spp. com outro produto a base de clorpirifós.

Quanto ao veículo de transporte do princípio ativo, verificou-se que tanto o óleo diesel quanto o querosene não afetaram a eficiência dos produtos termonebulígenos, indicando que qualquer um deles pode ser utilizado como veículo no processo. Anjos et al. (1998) relata que apenas a fumaça produzida pelo combustível do termonebulizador pode apresentar pequena eficiência de controle de sauveiros. Isso foi corroborado por Couto et al. (1978), que demonstraram que o óleo diesel apresentou apenas $25 \%$ de eficiência de controle de A. sexdens rubropilosa.

Pode-se concluir que todos os produtos testados apresentaram alta percentagem de paralisação das atividades de corte e de movimentação das formigas aos três e 12 dias após a aplicação, respectivamente. Os produtos Dursban e Dragnet (clorpirifós) e Attacid (extratos vegetais) apresentaram alta eficiência no controle de $A$. laevigata, sendo mais efetivos que a isca formicida MirexS Max.

Tabela 3 - Percentagem de paralisação de movimentação de formigas por dia após a aplicação dos tratamentos (DAA) e eficiência de controle de ninhos de Atta laevigata tratados com termonebulígenos e isca granulada. Três Marias, Minas Gerais (2000).

\begin{tabular}{|c|c|c|c|c|c|}
\hline \multirow[t]{2}{*}{ Tratamento } & \multicolumn{4}{|c|}{$\begin{array}{l}\text { Paralisação de movimentação de formigas (\%) } \\
\text { por DAA }\end{array}$} & \multirow{2}{*}{$\begin{array}{l}\text { Eficiência }(\%) \\
\text { (86 DAA) }\end{array}$} \\
\hline & 3 & 12 & 36 & 63 & \\
\hline Testemunha & $0 \mathrm{c}$ & $0 \mathrm{~b}$ & $0 \mathrm{~d}$ & $0 \mathrm{c}$ & $0 \mathrm{c}$ \\
\hline Dursban CE a $100 \mathrm{ml} /$ litro de diesel & $38 \mathrm{~b}$ & 88 a & $50 \mathrm{c}$ & $88 \mathrm{a}$ & $100 \mathrm{a}$ \\
\hline Dursban CE a $100 \mathrm{ml} /$ litro de querosene & $88 \mathrm{a}$ & $100 \mathrm{a}$ & $75 \mathrm{~b}$ & $50 \mathrm{~b}$ & $88 \mathrm{a}$ \\
\hline Dragnet CE a $140 \mathrm{ml} /$ litro de diesel & $13 \mathrm{~b}$ & $88 \mathrm{a}$ & $75 \mathrm{~b}$ & $100 \mathrm{a}$ & $100 \mathrm{a}$ \\
\hline Dragnet CE a $140 \mathrm{ml} /$ litro de querosene & $100 \mathrm{a}$ & $100 \mathrm{a}$ & $100 \mathrm{a}$ & $88 \mathrm{a}$ & $100 \mathrm{a}$ \\
\hline Attacid A a 200 ml/litro de diesel & $88 \mathrm{a}$ & $100 \mathrm{a}$ & $88 \mathrm{a}$ & $63 \mathrm{~b}$ & $100 \mathrm{a}$ \\
\hline Attacid B a $200 \mathrm{ml} /$ litro de diesel & $88 \mathrm{a}$ & $100 \mathrm{a}$ & $75 \mathrm{~b}$ & $50 \mathrm{~b}$ & $82 \mathrm{~b}$ \\
\hline Mirex-S MAX a $8 \mathrm{~g} / \mathrm{m}^{2}$ de formigueiro & $88 \mathrm{a}$ & $88 \mathrm{a}$ & $75 \mathrm{~b}$ & $63 \mathrm{~b}$ & $75 \mathrm{~b}$ \\
\hline
\end{tabular}

Médias seguidas pela mesma letra na coluna não diferem entre si (Tukey, p>0,05). 


\section{AGRADECIMENTOS}

Ao Conselho Nacional de Desenvolvimento Científico e Tecnológico (CNPq), à Coordenação de Aperfeiçoamento de Pessoal de Nível Superior (CAPES) e à Fundação de Amparo à Pesquisa do Estado de Minas Gerais (FAPEMIG).

\section{REFERÊNCIAS BIBLIOGRÁFICAS}

ALVES, J. B.; ZANUNCIO, J. C.; GALO, M. V.; ZANETTI, R. Paralisação de forrageamento e controle de Atta laevigata (Hymenoptera: Formicidae) com Mirex-S (sulfluramida) em duas metodologias de medição de formigueiros. Revista Árvore, Viçosa, v. 21, n. 1, p. 141146, 1997.

ALVES, J. B.; ZANUNCIO, J. C.; TORRES, J. B.; GALO, M. V. Métodos de distribuição de isca granulada em formigueiros de Atta laevigata (F. Smith). Revista Árvore, Viçosa, v. 20, n. 1, p. 111-116, 1996.

ANJOS, N.; DELLA LUCIA, T. M. C.; MAYHÉ-NUNES, A. J. Guia prático sobre formigas cortadeiras em reflorestamentos. Ponte Nova: Editora Graff Cor, 1998. 97 p.

COUTO, L.; ZANUNCIO, J. C.; ALVES, J. E. M.; CAMPINHOS, E.; SORESINI, L.; VARGAS, J. A. Avaliação da eficiência e custo do controle de Atta sexdens rubropilosa através do sistema termo-nebulizador, na região de Aracruz, ES. Revista Árvore, Viçosa, v. 2, n. 2, p. 8-16, 1978.

CRUZ, A. P.; ZANUNCIO, J. C.; ZANETTI, R.; GOMES, O. $\mathrm{S}$. Eficiência de iscas granuladas à base de sulfluramida $\mathrm{e}$ de clorpirifós no controle de Atta sexdens sexdens (Hymenoptera: Formicidae), no trópico úmido. Acta Amazônica, Manaus, v. 26, n. 3, p. 145-150, 1996.

CRUZ, J. M. da; NOGUEIRA, S. B.; PEREIRA, A. R.; NEUWES, B. O. Adaptação de uma motocicleta para termonebulização no controle de formigas saúvas (Atta spp.) em áreas reflorestadas de cerrado. Revista Árvore, Viçosa, v. 8, n. 2, p. 104-111, 1984.
DELLA LUCIA, T. M. C.; VILELA, E. F. Métodos atuais de controle e perspectivas. In: DELLA LUCIA, T. M. C. (Ed.). As formigas cortadeiras. Viçosa: UFV, 1993. p. 163-190.

GOMES, J. G.; MENDONÇA, N. T. de; PARMA, L. G. Controle de formigas cortadeiras do gênero Atta (formigas saúvas) com o inseticida clorpirifós em aplicação por termonebulização, com dois tipos de aparelhos. In: ENCONTRO DE MIRMECOLOGIA, 2., 1989, Viçosa. Anais... Viçosa: UFV, 1989. p. 22.

LARANJEIRO, A. J.; ZANUNCIO, J. C. Avaliação da isca à base de sulfluramida no controle de Atta sexdens rubropilosa pelo processo dosagem única de aplicação. IPEF, Piracicaba, v. 48/49, n. 1, p. 144-152, 1995.

SANTOS, A.; ZANETTI, R.; MENDONÇA, L. A.; MEDEIROS, A. G. B. Eficiência e custo de combate a ninhos de Atta spp. (Hymenoptera: Formicidae) com termonebulização. Cerne, Lavras, v. 13, n. 5, p. 23-27, 2007.

ZANETTI, R.; CARVALHO, G. A.; SANTOS, A.; SOUZASILVA, A.; GODOY, M. S. Manejo integrado de formigas cortadeiras. Lavras: UFLA, 2002. 16 p.

ZANETTI, R.; ZANUNCIO, J. C.; SOUZA-SILVA, A.; ABREU, L. G. Eficiência de isca formicida aplicada sobre o monte de terra solta de ninhos de Atta sexdens rubropilosa (Hymenoptera: Formicidae). Revista Árvore, Viçosa, v. 27, n. 3, p. 407-410, 2003.

ZANUNCIO, J. C.; COUTO, L.; SANTOS, G. P.; ZANUNCIO, T. V. Eficiência da isca granulada Mirex-S à base de sulfluramida, no controle de formiga cortadeira Atta laevigata (F. Smith, 1858) (Hymenoptera: Formicidae). Revista Árvore, Viçosa, v. 16, n. 3, p. $357-$ 361, 1992.

ZANUNCIO, J. C.; SOSSAI, M. F.; OLIVEIRA, H. N.; ZANUNCIO JUNIOR, J. S. Influência das iscas formicidas Mirex-S e Blitz na paralisação de corte e no controle de Atta sexdens rubropilosa (Hymenoptera: Formicidae). Revista Árvore, Viçosa, v. 26, n. 2, p. 237-242, 2002. 